\title{
Ligand-Free Noble Metal Nanocluster Catalysts on Carbon Supports via "Soft" Nitriding
}

\author{
Ben Liu ${ }^{\dagger}$, Huiqin Yao ${ }^{\dagger} \S$, Wenqiao Song ${ }^{\dagger}$, Lei Jin ${ }^{\dagger}$, Islam M. Mosa ${ }^{\dagger}$, James F.

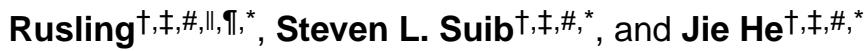

tDepartment of Chemistry, University of Connecticut, Storrs, Connecticut 06269, United States \#Institute of Materials Science, University of Connecticut, Storrs, Connecticut 06269, United States \#Green Emulsions, Micelles, and Surfactants Center, University of Connecticut, Storrs, Connecticut 06269, United States §Department of Chemistry, NingXia Medical University, Yinchuan 750004, China "Department of Surgery and Neag Cancer Center, University of Connecticut Health Center, Farmington, Connecticut 06232, United States "School of Chemistry, University of Ireland at Galway, Galway, Ireland

\section{Abstract}

We report a robust, universal "soft" nitriding method to grow in situ ligand-free ultrasmall noble metal nanocatalysts (UNMN; e.g., Au, Pd, and Pt) onto carbon. Using low-temperature urea pretreatment at $300{ }^{\circ} \mathrm{C}$, soft nitriding enriches nitrogen-containing species on the surface of carbon supports and enhances the affinity of noble metal precursors onto these supports. We demonstrated sub-2-nm, ligand-free UNMNs grown in situ on seven different types of nitrided carbons with no organic ligands via chemical reduction or thermolysis. Ligand-free UNMNs supported on carbon showed superior electrocatalytic activity for methanol oxidation compared to counterparts with surface capping agents or larger nanocrystals on the same carbon supports. Our method is expected to provide guidelines for the preparation of ligand-free UNMNs on a variety of supports and, additionally, to broaden their applications in energy conversion and electrochemical catalysis.

Supported noble metal particles ( $\mathrm{Au}, \mathrm{Pd}$, and $\mathrm{Pt}$ ) on high-surface-area supports are of great importance for many heterogeneous catalytic reactions. Numerous studies showed that catalytic activities of noble metal particles are highly size-dependent. In particular, ultrasmall noble metal nanocatalysts (UNMNs, $<2 \mathrm{~nm}$ ), i.e., nanoclusters with several to hundreds of atoms, have received substantial attention. They have a high surface-to-volume ratio and a large proportion of surface atoms, i.e. predominantly edge and corner atoms. ${ }^{1}$

\footnotetext{
*Corresponding Authors. james.rusling@uconn.edu (J.F.R.), steven.suib@uconn.edu (S.L.S.), jie.he@uconn.edu (J.H.). ASSOCIATED CONTENT

Supporting Information

The Supporting Information is available free of charge on the ACS Publications website at DOI: 10.1021/jacs.6b01702.
}

Synthetic details of ultrasmall and ligand-free noble metal NPs and carbon supports as well as additional electron microscopic images and electrocatalytic activity for methanol oxidation (PDF)

Notes

The authors declare no competing financial interest. 
Current manufacturing methods of supported noble metal particles, including impregnation and coprecipitation, lack size control. A broad size distribution of noble metal particles from a few nanometers to micrometers is usually obtained because of the weak affinity of noble metal precursors and/or their nanocatalysts to supports (carbon and oxides). ${ }^{2}$ Alternatively, UNMNs can be presynthesized and loaded onto solid supports. ${ }^{\text {1d,f }}$ The preparation of UNMNs has involved the use of excess capping agents to control sizes and nanostructures and to prevent overgrowth. ${ }^{3}$ However, surface capping agents often limit surface accessibility of UNMNs by blocking catalytic sites, and decrease activity and selectivity by interacting with intermediates. The removal of capping agents typically via the thermal deposition or oxidation (also known as activation) can lead to the sintering of UNMNs and loss of surface active sites. ${ }^{4}$ In view of fundamental interests and industrial applications, a facile, general synthetic method for supported ligand-free UNMNs is highly desirable.

This Communication reports a robust, green, general method to grow ligand-free UNMNs in situ onto carbon supports. We propose the concept of "soft" nitriding, similar to that used in steel processing, which is to diffuse nitrogen onto surfaces of carbon through low-

temperature annealing with urea $\left(300^{\circ} \mathrm{C}\right)$, while retaining the nanostructures and electronic properties of carbon supports. Soft nitriding introduces nitrogen-containing species to carbon surfaces through mild reaction with $\mathrm{NH}_{3}$ and $\mathrm{HCNO}$ generated from thermal decomposition of urea. Nitrogen-containing species can tremendously enhance the affinity of noble metal precursors onto carbon supports via electrostatic interactions. Using seven different activated carbon supports, we demonstrated that sub-2-nm, ligand-free, highly dispersed UNMNs can grow in situ on nitrided carbons in the absence of organic capping agents via chemical reduction or thermolysis. These carbon-supported, ligand-free UNMNs are exceptionally active for electrocatalytic methanol oxidation.

In a typical synthesis, nitrided carbon together with anionic metal precursors $\left(\mathrm{HAuCl}_{4}\right.$, $\mathrm{K}_{2} \mathrm{PdCl}_{4}$, or $\mathrm{K}_{2} \mathrm{PtCl}_{6}$ ) were dispersed in water (see Supporting Information (SI) for details). In situ chemical reduction of metal precursors was then carried out by adding $\mathrm{NaBH}_{4}$ to yield noble metal nanoclusters. Taking commercial Printex G carbon (PGC, Orion) loaded with Au nanocatalysts (AuNCs) as an example, the growth of AuNCs was confirmed by transmission electron microscopy (TEM). Figure 1a reveals that spherical PGC particles with an average diameter of $\sim 50 \mathrm{~nm}$ are uniformly covered with highly dispersed AuNCs (also see Figure S1). High-angle annular dark-field scanning TEM (HAADF-STEM) images show no aggregates or overgrowth of AuNCs (Figure 1b). AuNCs are uniform with an average diameter of $1.6 \pm 0.3 \mathrm{~nm}$ (Figure 1f). The high-resolution TEM (HR-TEM) image (Figure 1c) confirms the lattice fringes of $\mathrm{Au}$ (111) planes with $d$-spacings of $\sim 0.24 \mathrm{~nm}$ (only for larger clusters) and the graphitic layer (002) plane of carbon with $d$-spacings of $\sim 0.37 \mathrm{~nm}$. No wide-angle X-ray diffraction (XRD) peaks of Au can be seen, due to small particle size (Figure S2). STEM energy-dispersive X-ray spectroscopy (EDX) analysis shows that the bright nanodomains are Au phase, whereas dark domains are mainly $\mathrm{C}$ phase from PGC (Figure 1d). N elements are thoroughly distributed over the surface of nitrided PGC (N-PGC) (Figure S3). The loading amount of AuNCs in N-PGC/AuNCs is $2.34 \mathrm{wt} \%$ from atomic absorption spectroscopy, in agreement with that obtained from STEM-EDX mapping ( $2.3 \mathrm{wt} \%$ ) (Figure S4) and the feeding ratio of the $\mathrm{HAuCl}_{4}$ amount (2.4 wt \% by $\mathrm{Au}$ atoms). The high resolution X-ray photoelectron spectrum (XPS) of Au $4 \mathrm{f}$ displays 
asymmetric peaks at 83.2 and $87.0 \mathrm{eV}$, slightly lower than the reported value of Au films and Au clusters with thiol ligands, possibly due to the surface dipole generated by the N-PGC. The Brunauer-Emmett-Teller surface area of N-PGC/AuNCs is $29 \mathrm{~cm}^{2} \mathrm{~g}^{-1}$ (Figure S5), comparable to that of pristine PGC.

Control experiments further revealed the effect of soft nitriding. First, without the soft nitriding, the formation of much larger Au particles and aggregates occurred on the surface of PGC under identical reaction conditions (Figure S6). The likely reason is the absence of affinity of $\mathrm{AuCl}_{4}{ }^{-}$on the surface of PGC, where Au particles were reduced within the solution and then physically adsorbed on carbons, as in previous studies. ${ }^{5}$ Second, we examined six other commercial or homemade carbons, including Printex U (Orion), OLC Coconut Shell AC (12-30 mesh, Calgon), activated charcoal (100-400 mesh, Sigma), Vulcan XC-72 (FuelCellStore), mesoporous carbon film, and mesoporous carbon nanosphere, to confirm the importance of soft nitriding. All urea-treated carbons supported in situ growth of ultrasmall AuNCs, regardless of carbon nanostructures and surface areas (see Figures S7-12). The average size of AuNCs is in the range 1-2 nm, similar to that obtained on N-PGCs. The initial surface chemistries and topologies of carbon supports had minimum impact on the growth of ultrasmall AuNCs.

XPS was first used to confirm the change in elemental compositions after soft nitriding. The surface of PGC presented 16 atom \% of oxygen and no detectable N, while the N-level was estimated to be 19 atom \% after soft nitriding (Figure 2a), much higher than that obtained using other methods. ${ }^{6}$ High-resolution $\mathrm{C}$ 1s spectra showed both samples have a similar carbon composition at $\sim 284 \mathrm{eV}$, assigned to graphitic $\mathrm{sp}^{2}$ carbon (Figure $2 \mathrm{~b}$ and Figure S14 for N-GPC/AuNCs). A slight decrease of binding energy $(\sim 0.7 \mathrm{eV})$ for N-PGC can be seen, due to reduction of surface carbon. There is a stronger shoulder at $289 \mathrm{eV}$ for N-PGCs, corresponding to a higher percentage of $\mathrm{sp}^{3}$ carbon with $\mathrm{C}-\mathrm{O}$ and $\mathrm{C}-\mathrm{N}$ bonds. ${ }^{7}$ The $\mathrm{N} 1 \mathrm{~s}$ XPS spectrum of N-PGC fit well onto a three-peak model (Figure 2c). A large percentage $(68 \%)$ of the $\mathrm{N}$ atoms have a binding energy of $400 \mathrm{eV}$, indexed to amine and amide groups, e.g. C-NH and $\mathrm{C}-\mathrm{NH}_{2}$. The peak with a higher binding energy at $401.3 \mathrm{eV}(17 \%)$ was attributed to quaternary $\mathrm{N}$ atoms that replaced carbon atoms in the graphitic surface layers. The peak with a lower binding energy at $398.1 \mathrm{eV}(15 \%)$ was assigned to the pyridine N. ${ }^{6 a, 7}$ The nitriding into the graphitic layers occurred in both of the last two scenarios. The nitrogen doping in graphitic layers can enhance the crystallinity of graphitic carbon as confirmed by wide-angle XRD where a sharp peak appeared at $2 \theta=27.8^{\circ}$ (Figures S15-16).

We investigated the variation of surface functional groups using ${ }^{1} \mathrm{H}$ NMR and Fourier transform infrared (FT-IR) spectroscopy. ${ }^{1} \mathrm{H}$ NMR spectra of PGCs in $d_{6}$-dimethyl sulfoxide (DMSO) before and after soft nitriding are shown in Figure 2d (see Figure S17 for ${ }^{1} \mathrm{H}$ NMR spectra at different annealing times). No detectable proton signals are present in PGC, while well-resolved proton peaks appear in N-PGC. The two peaks at 10.8 and $6.6 \mathrm{ppm}$ can rapidly exchange with $\mathrm{D}_{2} \mathrm{O}$ and are assigned to $\mathrm{NH}$ and $\mathrm{NH}_{2}$ of ureido groups, respectively (see Figure S18 for deuterium exchange spectra). This agrees with XPS spectra where the majority of $\mathrm{N}$ is in $\mathrm{C}-\mathrm{NH}$ and $\mathrm{C}-\mathrm{NH}_{2}$. Surface carboxylic and hydroxyl groups of activated carbons are solely responsible for formation of these ureido groups via the addition reaction of HNCO. ${ }^{8}$ The sharp peak at $10.4 \mathrm{ppm}$, also exchangeable with $\mathrm{D}_{2} \mathrm{O}$, is assigned to the $\mathrm{NH}$ 
of primary ketimine groups originating from the reduction of carboxyls with $\mathrm{NH}_{3}$. Other peaks are nonexchangeable with $\mathrm{D}_{2} \mathrm{O}$ and are assigned to protons on reduced $\mathrm{sp}^{3}$ and $\mathrm{sp}^{2}$ carbon atoms. For example, the peak at $2.9 \mathrm{ppm}$ can be assigned to $\mathrm{HC}-\mathrm{N}$ where defect sites on PGCs were reduced, accompanying incorporation of $\mathrm{N}$ atoms. Meanwhile, the presence of these functional groups after soft nitriding was also confirmed by FT-IR spectra (Figure 2e). Broad peaks at 3360 and $1509 \mathrm{~cm}^{-1}$ are assigned to the stretching and bending of $\mathrm{H}-\mathrm{N}$ from ureido groups, respectively, while, the strong peaks at 1812 and $1757 \mathrm{~cm}^{-1}$ are ascribed to the stretching of $\mathrm{C}=\mathrm{O}$ groups of ureido groups. Raman spectra show similar $\mathrm{G}$ and $\mathrm{D}$ bands for PGC after soft nitriding, suggesting that the electronic properties of carbon are unaltered (Figure S19).

Based on the above-mentioned results, we deduced that soft nitriding occurred mainly via chemical modifications of oxygen-containing sites on the surface of activated carbons. Thermolysis products of urea, i.e. $\mathrm{HNCO}$ and $\mathrm{NH}_{3}$, integrate nitrogen-containing species into the carbon supports at mild temperatures. The two reaction pathways of $\mathrm{HNCO}$ and $\mathrm{NH}_{3}$ seem to be independent to yield ureido groups and $\mathrm{N}$-doping on graphitic layers, respectively. Soft nitriding preserved ureido groups, unlike the $\mathrm{NH}_{3}$-reduction process solely. Both ureido groups and nitrogen-doping on the graphite frameworks lead to (i) strong affinity to anionic metal precursors and (ii) stabilization of AuNCs to avoid overgrowth.

We also examined the growth of metal clusters by varying precursors and the $\mathrm{pH}$ of the solution. First, the loading of nanocatalysts did not take place when using cationic metal precursors of group 10 and 11 elements, e.g. $\mathrm{Pd}^{2+}, \mathrm{Cu}^{2+}$, and $\mathrm{Ag}^{+}$. The nucleation of nanocatalysts on the nitrided carbon supports therefore is somewhat dependent on electrostatic interactions. Second, the growth of AuNCs can be modulated by the $\mathrm{pH}$ of the growth solution (Figure S21). Ultrasmall AuNCs grew on N-PGCs in a pH range of 4-10. However, at $\mathrm{pH}<4$ or $>10$, only larger Au particles with an average diameter of 6-10 nm were obtained. Therefore, graphitic nitrogen species, including quaternary and pyridinic $\mathrm{N}$ atoms on the surface of carbon, can be responsible for interaction of metal precursors with nitrided carbon, as well as the nucleation of AuNCs. Moreover, the balance of the nucleation rate and electrostatic interaction of precursors/supports is crucial to control the size of AuNCs. The nucleation rate of $\mathrm{AuCl}_{4}{ }^{-}$is much faster at lower $\mathrm{pH} .{ }^{9}$ Nucleation and growth of AuNCs took place in solution rather than on the surface of carbon at $\mathrm{pH}<3$, due to fast nucleation. At $\mathrm{pH}>10$, the low affinity of $\mathrm{AuCl}_{4}{ }^{-}$to the carbon limited the nucleation and growth of Au particles on N-PGCs.

Our synthetic approach allows tuning of the loading amount of UNMNs by simply varying feeding ratios of metal precursors (relative to carbon). Five different loading amounts of AuNCs ( 0.25 to $10 \mathrm{wt} \%)$ were demonstrated on N-PGC (Figure S22). Well-defined AuNCs with an average diameter of $0.7-2 \mathrm{~nm}$ were produced, although smaller particles tended to form at lower loadings of AuNCs. However, aggregates of Au particles ( $>3 \mathrm{~nm})$ appeared when the loading amount reached $>10 \mathrm{wt} \%$. Moreover, other catalytically active UNMNs can grow on nitrided carbons using a similar procedure. Figure 3 shows the ultrasmall PdNCs and PtNCs loaded on N-PGCs with $\mathrm{K}_{2} \mathrm{PdCl}_{4}$ and $\mathrm{K}_{2} \mathrm{PtCl}_{4}$ as precursors. The average diameters of PdNCs and PtNCs are $1.4 \pm 0.2$ and $1.5 \pm 0.3 \mathrm{~nm}$, respectively. 
We emphasize that chemical reduction to yield UNMNs is uncompelled. Thermolysis of noble metal precursors on nitrided carbon supports can produce comparable results (similar to impregnation; see details in SI and TEM images in Figure S23). Gram-level production was validated using thermolysis. For mass production of industrial catalysts, this may be more cost-effective for scale-up.

Electrocatalytic methanol oxidation was finally chosen as a model reaction to evaluate the activity of ligand-free UNMNs supported on carbon. Negatively charged PGC/AuNCs were first loaded on pyrolytic graphite disk electrodes via electrostatic layer-by-layer deposition (see SI for details). Two thiolated AuNCs with 3-mercaptopropionic acid and dodecanethiol (denoted as AuNCs-MPA and AuNCs-DT) and PGC loaded with larger Au particles (6 nm, obtained at $\mathrm{pH}=3$ ) were used as controls. Figure 4a depicts typical cyclic voltammograms (CVs) of $1 \mathrm{M}$ methanol in $0.1 \mathrm{M} \mathrm{NaOH}$ at a scan rate of $10 \mathrm{mV} / \mathrm{s}$ (see Figure S25 for other scan rates). Ligand-free AuNCs showed the highest peak current density of $1.19 \mathrm{~A} / \mathrm{mg}_{\mathrm{Au}}$ and the lowest oxidation peak potential of $1.11 \mathrm{~V}$ on the positive (anodic) scan. When capped with surface ligands, both AuNCs-MPA and AuNCs-DT gave a $100 \mathrm{mV}$ higher peak potential of $1.21 \mathrm{~V}$ than that of ligand-free AuNCs. Also, peak current density decreased to 560 and $470 \mathrm{~mA}$ per $\mathrm{mg}_{\mathrm{Au}}$, corresponding to a $53 \%$ and $61 \%$ loss of the mass activity (Figure 4c), respectively. Ligand-free AuNCs displayed much higher catalytic activity with a turnover frequency (TOF) of $0.59 \mathrm{~s}^{-1}, 2.1$ and 2.5 times higher than that of AuNCs-MPA and AuNCs-DT (Table S2). The heterogeneous electrochemical rate constant for methanol oxidation of the ligand-free AuNCs of $\sim 0.02 \mathrm{~cm} / \mathrm{s}$ was $\sim 3$-fold larger than that of AuNCsMPA and AuNCs-DT (Figure S27 and Table S1). An even more dramatic ligand effect was found for ligand-free PdNCs with an anodic current density of $1.9 \mathrm{~A} / \mathrm{mg}_{\mathrm{Pd}}$ at a peak potential of $0.80 \mathrm{~V}, 38$ times more active compared to that of PdNCs capped by DT, which in this case almost completely shuts down catalytic oxidation (Figure $4 b$ ). The dramatic decrease in methanol oxidation performance for PtNCs with ligands possibly originates from the formation of a PdS interlayer which blocks catalytic sites of PtNCs. ${ }^{10}$ The surface ligands block catalytic sites and decrease electrocatalytic activity dramatically. Moreover, the size effect of AuNCs was also examined using ligand-free $6 \mathrm{~nm}$ AuNC-PGCs. A positive shift of the oxidation peak potential to $1.23 \mathrm{~V}$ and decrease in peak current density to 380 $\mathrm{mA} / \mathrm{mg}_{\mathrm{Au}}$ were found (Figure 4a).

In summary, we report a new, facile, environmental friendly soft nitriding technique to load ligand-free noble metal nanoclusters onto virtually any carbon supports. Soft nitriding enriched the nitrogen-containing species on the surface of carbon via low-temperature urea annealing. Sub-2-nm, ligand-free UNMNs were grown in situ directly on nitrided carbons in the absence of any ligands. Nitrogen-containing species enhanced the affinity of supports for metal precursors/nanocrystals, thus preventing aggregation and overgrowth of nanocrystals. Moreover, ligand-free UNMNs on carbon exhibited far better electrocatalytic activities for methanol oxidation, compared to counterparts with surface capping agents and/or larger nanocrystals. Our synthetic method is highly reproducible and easy to scale up. Our results are expected to provide useful guidelines to manufacture supported noble metal nanoclusters and to selectively access the ligand effect in nanocatalysis. 


\section{Supplementary Material}

Refer to Web version on PubMed Central for supplementary material.

\section{Acknowledgments}

J.H. thanks financial support from the University of Connecticut. S.L.S. acknowledges support of the US Department of Energy, Office of Basic Energy Sciences, Division of Chemical, Biological and Geological Sciences under Grant DEFG02-86ER13622.A000. TEM studies were performed using facilities of UConn/FEI Center for Advanced Microscopy and Materials Analysis (CAMMA). This work was also partially supported by the Green Emulsions Micelles and Surfactants (GEMS) Center, FEI Company under an FEI-UConn partnership agreement, a UConn Research Excellence Program Award, and National Institute of Environmental Health Sciences, NIH, Grant No. ES03154 (J.F.R.).

\section{REFERENCES}

1. (a) Ertl G. Angew. Chem., Int. Ed. 2008; 47:3524.(b) Hutchings GJ, Kiely CJ. Acc. Chem. Res. 2013; 46:1759. [PubMed: 23586905] (c) Sun, J-k; Zhan, W-w; Akita, T.; Xu, Q. J. Am. Chem. Soc. 2015; 137:7063. [PubMed: 26020572] (d) Turner M, Golovko VB, Vaughan OP, Abdulkin P, Berenguer-Murcia A, Tikhov MS, Johnson BF, Lambert RM. Nature. 2008; 454:981. [PubMed: 18719586] (e) Huang J, Akita T, Faye J, Fujitani T, Takei T, Haruta M. Angew. Chem., Int. Ed. 2009; 48:7862.(f) Li G, Jin R. Acc. Chem. Res. 2013; 46:1749. [PubMed: 23534692] (g) Tsunoyama H, Sakurai H, Negishi Y, Tsukuda T. J. Am. Chem. Soc. 2005; 127:9374. [PubMed: 15984857]

2. Haruta M. J. New Mater. Electr. Sys. 2004; 7:163.

3. (a) Niu Z, Li Y. Chem. Mater. 2014; 26:72.(b) Liu Y, Tsunoyama H, Akita T, Xie S, Tsukuda T. ACS Catal. 2011; 1:2.

4. Cargnello M, Chen C, Diroll BT, Doan-Nguyen VV, Gorte RJ, Murray CB. J. Am. Chem. Soc. 2015; 137:6906. [PubMed: 25961673]

5. (a) Rolison DR. Science. 2003; 299:1698. [PubMed: 12637736] (b) Yan S, Zhang S, Lin Y, Liu G. J. Phys. Chem. C. 2011; 115:6986.

6. (a) Wang X, Li X, Zhang L, Yoon Y, Weber PK, Wang H, Guo J, Dai H. Science. 2009; 324:768. [PubMed: 19423822] (b) Liang H-W, Zhuang X, Brüller S, Feng X, Müllen K. Nat. Commun. 2014; 5:4973. [PubMed: 25229121]

7. Li X, Wang H, Robinson JT, Sanchez H, Diankov G, Dai H. J. Am. Chem. Soc. 2009; 131:15939. [PubMed: 19817436]

8. Ford WE, Jung A, Hirsch A, Graupner R, Scholz F, Yasuda A, Wessels JM. Adv. Mater. 2006; 18:1193.

9. Ji X, Song X, Li J, Bai Y, Yang W, Peng X. J. Am. Chem. Soc. 2007; 129:13939. [PubMed: 17948996]

10. Vericat C, Vela M, Corthey G, Pensa E, Cortés E, Fonticelli M, Ibañez F, Benitez G, Carro P, Salvarezza R. RSC Adv. 2014; 4:27730. 

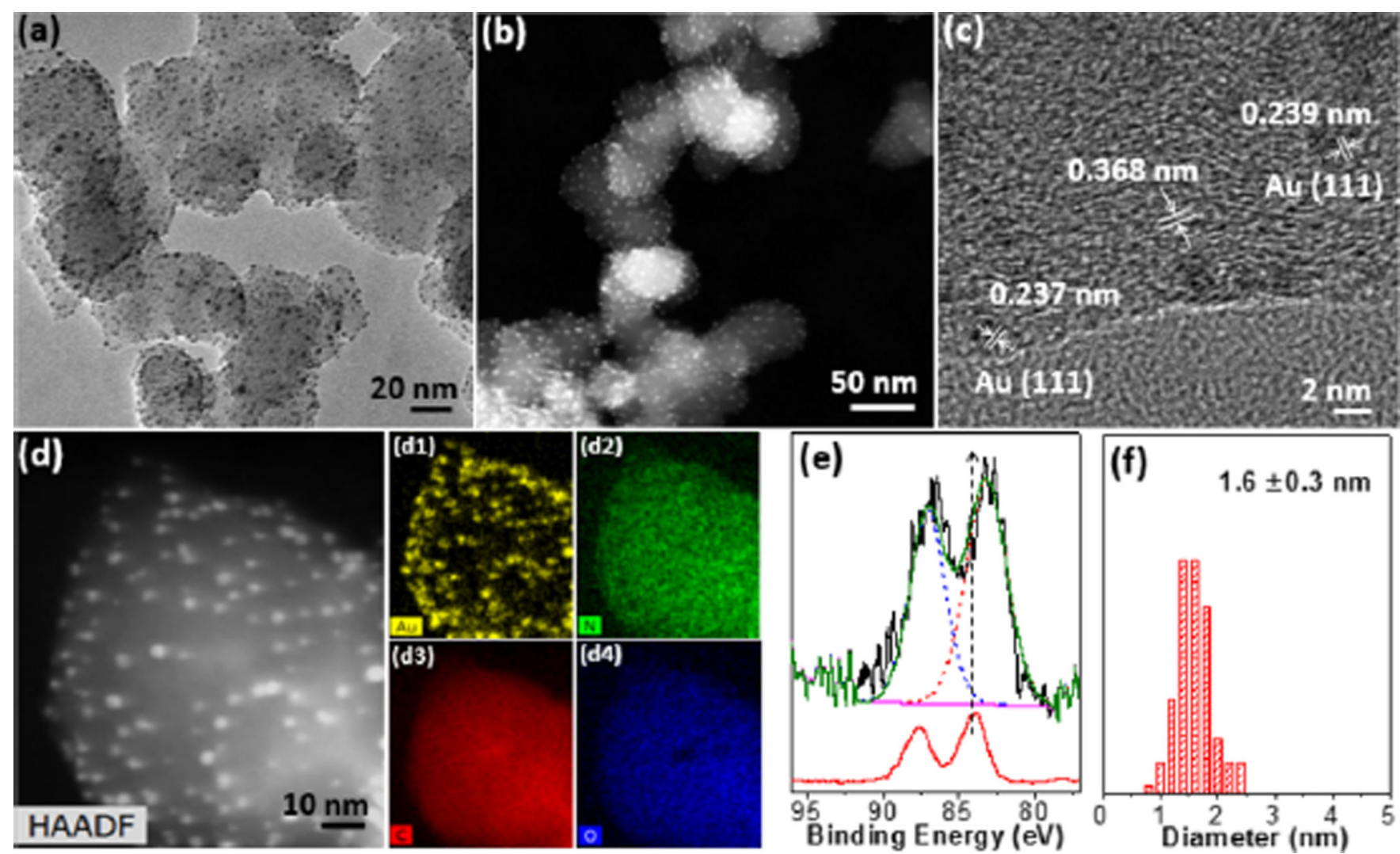

Figure 1.

(a) Bright-field TEM, (b) HAADF-STEM, (c) HR-TEM images, and (d) STEM EDX mappings of N-PGC/AuNCs: Au, N, C, and O are given in (d1-d4), respectively. (e) Highresolution XPS Au $4 \mathrm{f}$ spectrum of N-PGC/AuNCs. The bottom spectrum (red) is from $\mathrm{Au}$ thin film. (f) The average diameter and distribution of AuNCs. 

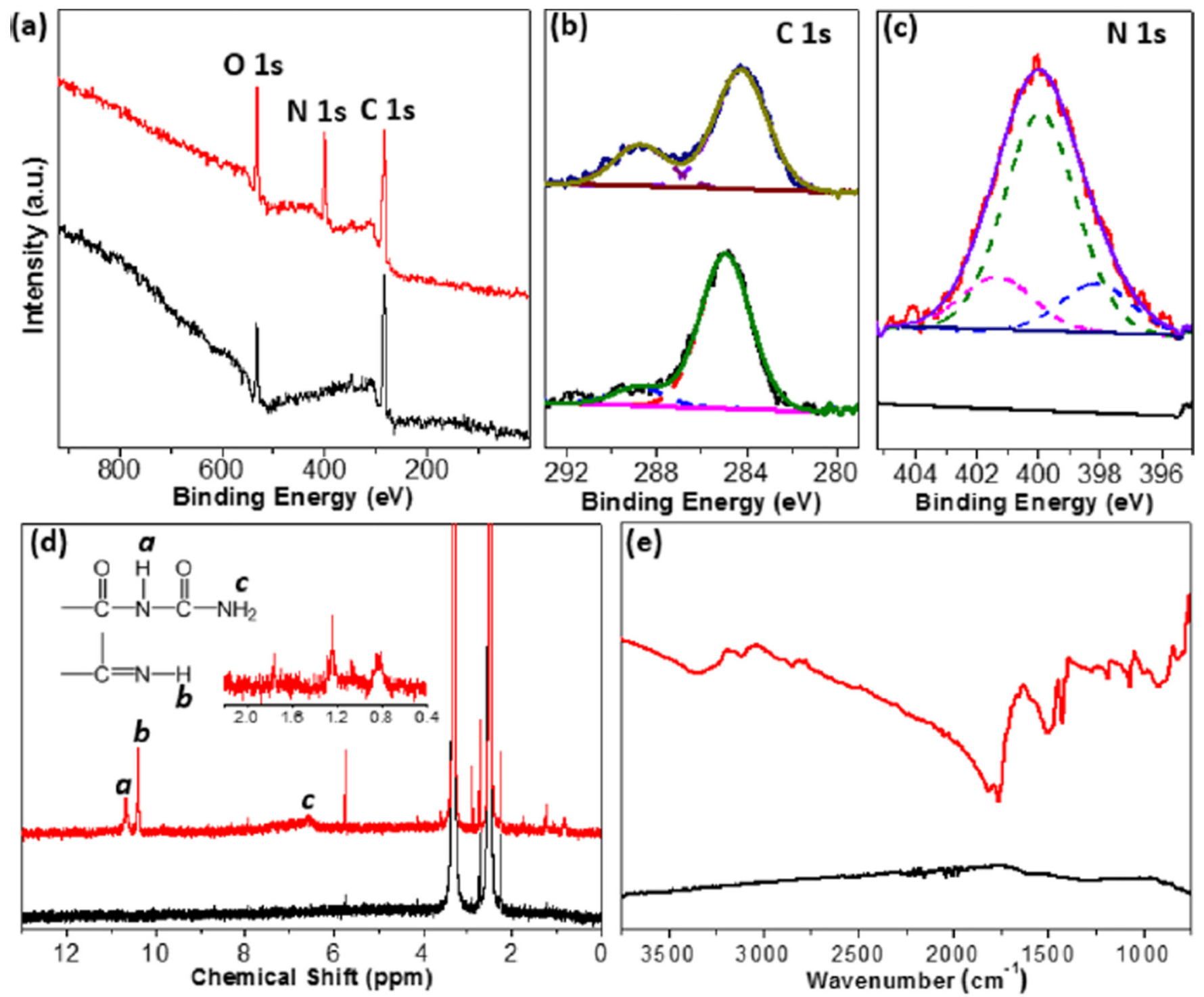

Figure 2.

Survey XPS spectra (a), high-resolution C 1s and N 1s XPS spectra (b-c), ${ }^{1} \mathrm{H}$ NMR spectra in $d_{6}$-DMSO (d), and FT-IR spectra of PGCs (bottom) and N-PGCs (top) (e). The inset in (d) is a zoom-in spectrum of N-PGCs. 

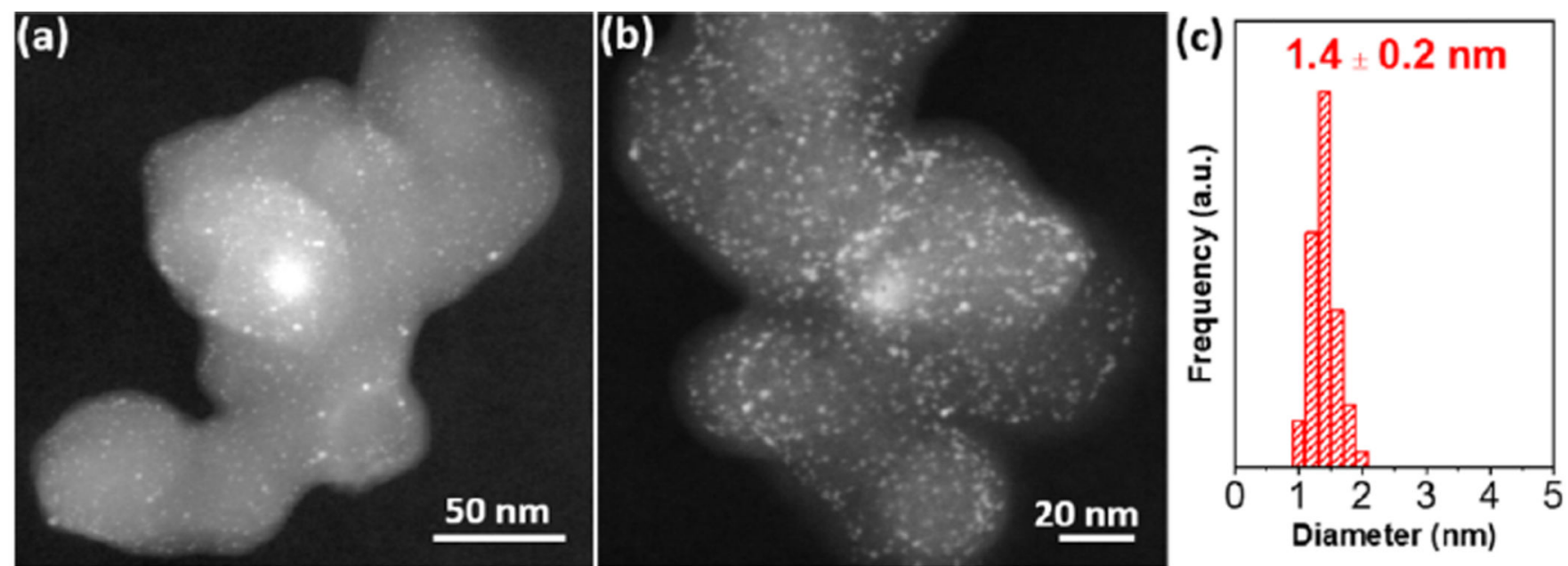

를
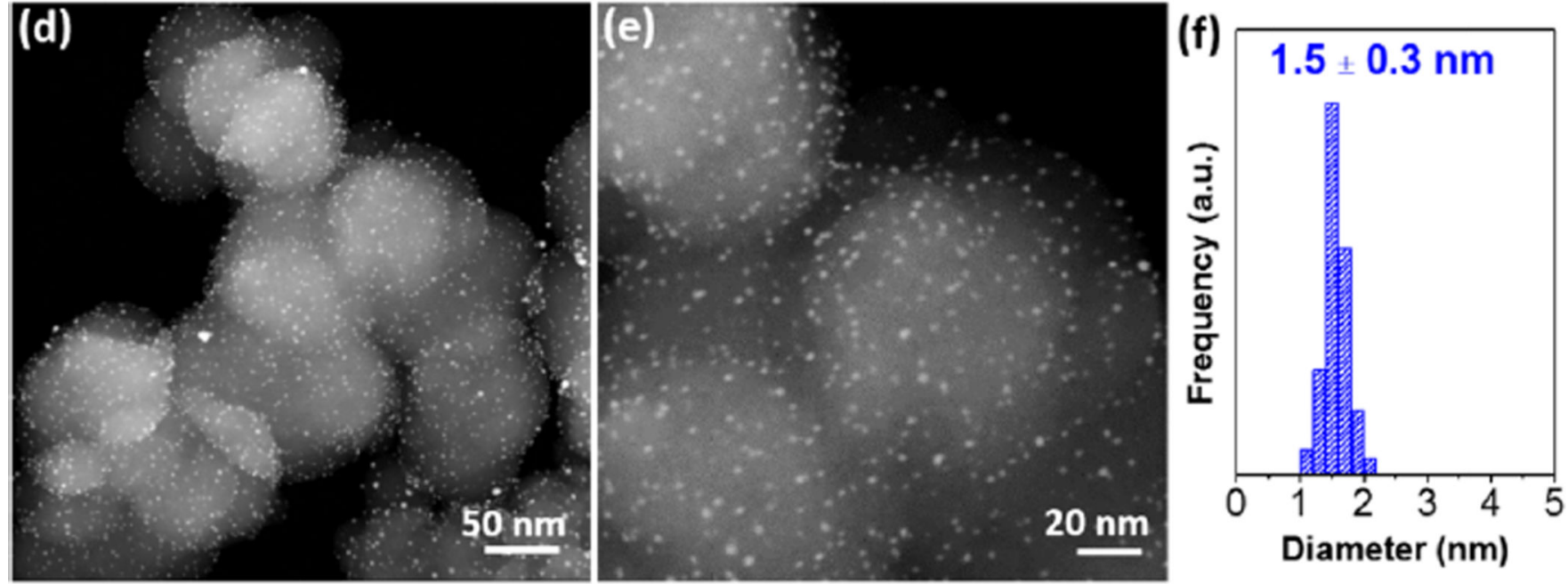

Figure 3.

HAADF-STEM images and size distributions of PdNCs $(\mathrm{a}-\mathrm{c})$ and PtNCs $(\mathrm{d}-\mathrm{f})$ prepared on N-PGCs. Loading amount of PdNCs and PtNCs is 2.4 and $2.1 \mathrm{wt} \%$, respectively. 

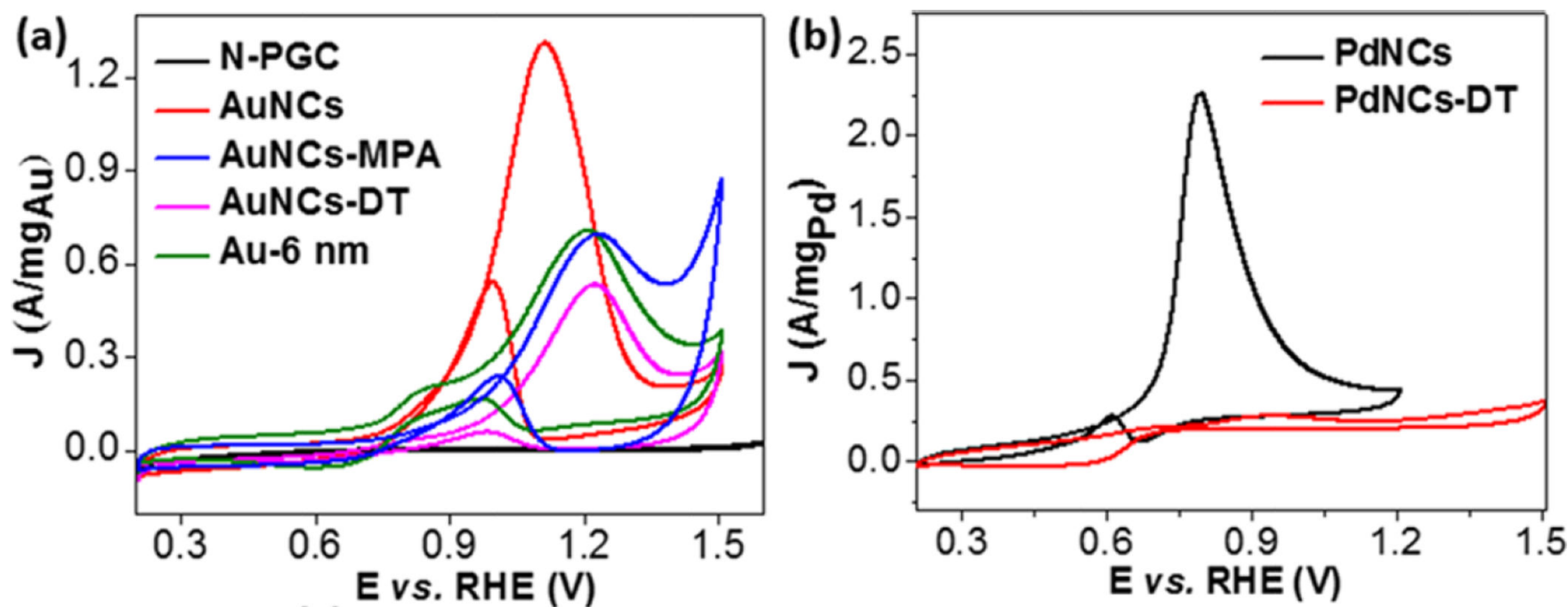

(c)

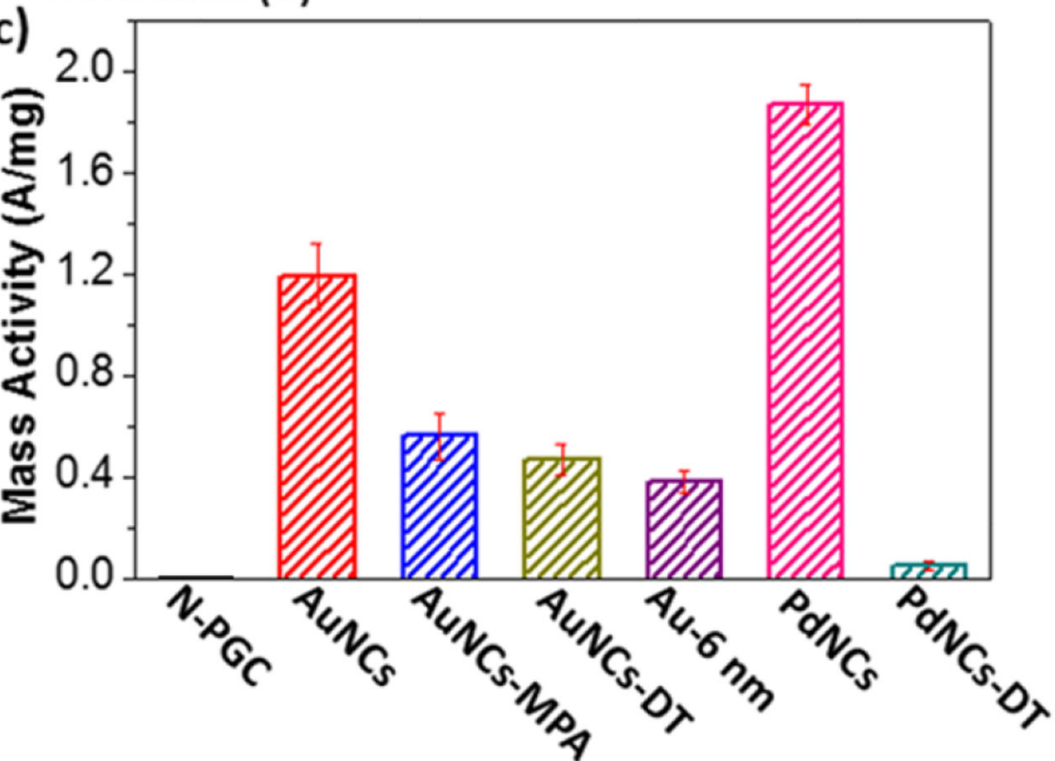

Figure 4.

Electrocatalytic oxidation activities of UNMNs reflected by CVs for $1 \mathrm{M}$ methanol in $0.1 \mathrm{M}$ $\mathrm{NaOH}$. (a) CVs of N-PGC, N-PGC/AuNCs, N-PGC/AuNCs-MPA, N-PGC/AuNCs-DT, and N-PGC/Au-6 nm; (b) CVs of N-PGC/PdNCs and N-PGC/PdNCs-DT. The CV curve of NPGC was normalized to the weight of carbon, while other curves were normalized to the weight of metal clusters. (c) Mass activities of UNMNs at the peak potentials. The mass activities in (c) were averaged from three independent measurements. 